\title{
References.
}

1. Gross-Tsur, et al. J Pediatr. 1997 Apr;130(4):670-4.

2. Hemmer SA, et al. Pediatr Neurol. 2001 Feb;24(2):99-102.

\section{RISK FACTORS BY GENDER FOR CHILDREN WITH ADHD}

Investigators at University of Western Australia conducted a population-based, case-control study of maternal, pregnancy, and newborn risk factors by gender for children prescribed stimulant medication for treatment of ADHD. Mothers of children with ADHD were significantly more likely to be younger, be single, have smoked in pregnancy, have labor induced, and experience threatened preterm labor, preeclampsia, urinary tract infection in pregnancy, or early term delivery irrespective of the gender of the child, compared with the control group. A possible protective effect of oxytocin augmentation is noted in girls. Factors not identified as risk factors included low birth weight, postterm pregnancy, small for gestational age infant, fetal distress, and low Apgar scores. (Silva D, Colvin L, et al. Environmental risk factors by gender associated with attention-deficit/hyperactivity disorder. Pediatrics 2014 Jan;133(1):e14-22).

COMMENTARY. Evidence of environmental mediators in ADHD is demonstrated in twin studies, affected twins having greater exposure to certain risk factors [1]. A familial, genetic factor in an estimated $80 \%$ of cases may involve the dopamine receptor and transporter genes, but gene-environment interaction is increasingly recognized as a mechanism in the etiology of ADHD [2][3]. Environmental factors may occur prenatally, in the perinatal period, or postnatally [4]. Of all the environmental factors implicated, maternal smoking and nicotine exposure attract the most attention in the literature, but in practice, maternal cigarette smoking is almost invariably denied [4].

\section{References.}

1. Lehn H, et al. J Am Acad Child Adolesc Psychiatry. 2007 Jan;46(1):83-91.

2. Thapar A, et al. Br J Psychiatry. 2007 Jan;190:1-3.

3. Ficks CA, Waldman ID. Curr Psychiatry Rep. 2009 Oct;11(5):387-92.

4. Millichap JG. Pediatrics. 2008 Feb;121(2):e358-65.

\section{NEUROMUSCULAR DISORDERS}

\section{AXONAL NEUROPATHY, MICROCEPHALY AND VRK1 MUTATIONS}

Investigators from Baylor College of Medicine, Texas Children's Hospital, Houston, TX; Kennedy Krieger Institute, Baltimore, MD; and University of Minnesota, $\mathrm{MN}$, report 3 patients from 2 unrelated families with a complex neuropathy phenotype characterized by axonal sensorimotor neuropathy, severe nonprogressive microcephaly and cerebral dysgenesis. Compound heterozygous alleles responsible for the clinical phenotype were identified by whole-genome and whole-exome sequencing in 2 affected siblings from 1 family and a homozygous nonsense variant in the third unrelated patient 
in the vaccinia-related kinase 1 gene (VRK1). (Gonzaga-Jauregui C, Lotze T, Jamal L, et al. Mutations in VRK1 associated with complex motor and sensory axonal neuropathy plus microcephaly. JAMA Neurol 2013 Dec;70(12):1491-8).

COMMENTARY. Hereditary motor and sensory neuropathies (HMSNs) are a group of slowly progressive diseases genetically heterogeneous, with more than 40 disease-associated genes identified. VRK1 is a novel HMSN locus that can be associated with a complex peripheral neuropathy phenotype, an autosomal recessive axonal motor sensory neuropathy and microcephaly. Genome-wide analysis enables the identification of novel HMSN-associated genes.

\section{AXONAL NEUROPATHY WITH NEUROMYOTONIA}

Investigators from the Children's Hospital, Coimbra, Portugal, and centers in Belgium, report a 16-year-old girl with consanguineous parents who presented with progressive distal muscular atrophy and weakness, beginning at age 6 years. After 10 years follow-up, clinical myotonia developed and was confirmed by electrophysiologic studies. This severe chronic motor axonal neuropathy was associated with a homozygous mutation in HINT1 and with late onset neuromyotonia. Sensory impairment was discrete and also appeared late. (Caetano JS, Costa C, Baets J, et al. Autosomal recessive axonal neuropathy with neuromyotonia: A rare entity. Pediatr Neurol 2014 Jan;50(1):104-7).

COMMENTARY. Autosomal recessive axonal neuropathy with neuromyotonia is recently described in 50 patients from 33 families, with 8 different HINT1 mutations, in a report including the above patient and cited by the authors [1]. The disease presents in the first decade with distal muscle weakness in upper and lower limbs. Sensory impairment is mild in some and most develop action myotonia in the hands and orthopedic deformities.

\section{References.}

1. Zimon M, et al. Nat Genet. 2012 Oct;44(10):1080-3.

\section{LAMBERT-EATON SYNDROME IN CHILDREN}

Investigators from Boston Children's Hospital; the Lahey Clinic, Burlington, MA; and Ohio State University, report 3 children presenting between ages 9 and 10 years with proximal lower extremity weakness with areflexia and low-amplitude compound muscle action potentials, and diagnosed with Lambert-Eaton myasthenic syndrome. A literature review found 9 other pediatric cases of Lambert-Eaton myasthenic syndrome, 3 having associated malignancies, 2 with lymphoproliferative disorders and 1 with neuroblastoma. The 9 nonparaneoplastic patients with primary autoimmune disorder responded to immunomodulatory therapy, with complete remission in 2 patients. (Hajjar M, Markowitz J, Darras BT, Kissel JT, Srinivasan J, Jones HR. Lambert-Eaton syndrome, an unrecognized treatable pediatric neuromuscular disorder: Three patients and literature review. Pediatr Neurol 2014 Jan;50(1):11-7). 